\title{
Editorial of CCF transactions on networking: special issue on edge computing and networking
}

\author{
Dan Wang ${ }^{1} \cdot{\text { Fangming } \mathrm{Liu}^{2} \cdot \text { Wei Bao }^{3} \cdot \text { Lin Wang }^{4} \cdot \text { Jiangchuan Liu }}^{5} \cdot$ Yuanyuan Yang $^{6} \cdot$ Weisong Shi $^{7}$
}

Published online: 3 May 2019

(c) China Computer Federation (CCF) 2019

\section{Introduction}

The success of the cloud is phenomenal in the last decade; however, researchers and practitioners start to realize that the cloud itself has limitations in supporting many emerging applications in the last few years. The proliferation of Internet of Things (IoT), the large amount of data generated, the wide diversity of the applications, and the cost of transmitting all data to the cloud, have pushed the horizon of a new computing paradigm, Edge Computing, which calls for processing the data at the edge of the network. Edge computing has the potential to address the concerns of response time requirement, battery life constraint, bandwidth cost saving, as well as data safety and privacy. The applications and research opportunities related to Edge Computing are immense. From applications point of view, there are emerging applications in video analytics, smart environments, autonomous driving, mobile sensing, industry 4.0, collaborative games, and logistics. From the viewpoint of technologies, there are challenges in edge computing architecture, resource management, data sensing, sharing and processing, quality of services, and networking protocols. From the viewpoint of foundations, edge computing requests for new advances in wireless communication, parallel and distributed computing, network economics, computer architecture,

Dan Wang

csdwang@comp.polyu.edu.hk

1 The Hong Kong Polytechnic University, Hung Hom, Hong Kong

2 Huazhong University of Science and Technology, Wuhan, China

3 University of Sydney, Sydney, Australia

TU Darmstat, Darmstat, Germany

5 Simon Fraser University, Burnaby, Canada

6 Stony Brook University, Stony Brook, USA

7 Wayne State University, Detroit, USA as well as security and privacy understanding. This special issue is meant to provide a platform for researchers with different expertise to discuss Edge Computing.

\section{Papers in this special issue}

In this special issue, we select a total of six papers. They represent four important research directions in edge computing: (1) new framework; a new framework is necessary to support a set of applications with common abstractions. A particular set of applications is AI applications. In the past, AI applications were process in the cloud because the data are in the cloud, e.g., the recommendation systems. Nowadays, there is an increasing number of AI applications where data are generated at the edge. This requests for new frameworks to ease the development and deployment. We have one representative paper in this category that discusses an edge framework for deep learning applications; (2) applying edge computing to various applications; for many applications, it is neither architecturally correct, nor resource efficient to execute the application in the cloud. With the recent advances and acceptance of edge computing, research start to study how to utilize edge computing to serve applications, and the problem within. We have two representative papers in this category that discuss vehicle localization and mobile crowdsourcing using edge computing respectively; (3) edge management; clearly it will take some time for the edge to function smoothly, as what we have learned from the Internet and the cloud computing in the past. There will be conflicts between sub systems, and resource utilization management. We have two papers in this category that discuss the conflict between C-RAN and Mobile Edge Computing, and cache management respectively; and (4) security and privacy; security and privacy are always and important problems. Edge systems are no exception. We have one representative paper in this category that shows that anonymous 
mobility traces are vulnerable to asynchronous side information attack from the edge.

\subsection{New framework}

The paper "Exploiting the Edge Power: An Edge Deep Learning Framework" presents an edge computing framework for supporting deep learning applications. The paper argues that crowdsourced big data from Internet users have long been of interest to modern machine learning technologies, and recent advances in deep learning have shown great potentials in exploring the hidden information therein. Deep learning relies on strong computation power to process the massive amount of data, which is typical offered by modern data centers, as well as the data storage. A cloud built on top of the data center, which seamlessly integrates storage and computation, seems to be an ideal platform for learning. It however faces significant challenges from data collection and service distribution over the network, given the end users are globally and remotely distributed. This paper presents edge learning for networked intelligent applications, which complements the cloud centric design to effectively reduce network traffic and inference latency. The paper discusses the key design issues of edge learning, including strategies to push data preprocessing and preliminary learning to the network edge, as well as to confine computation to local regions with high accuracy. A prototype demonstrates its feasibility with off-the-shelf hardware and confirms its superiority with real world experiments.

\subsection{Applying edge computing to various applications}

The paper "Edge-empowered Accurate Urban Vehicle Localization with Cellular-Aware Trajectories" presents a case of using edge computing to improve the accuracy of vehicle location information in urban settings. This is a challenging problem due to the complexity of urban environments. In this paper, a novel scheme, called Urban Positioning System (UPS), is proposed. The key observation comes from extensive empirical study, where the authors found that GSM power spectrogram collected over a distance has ideal temporal-spatial characteristics for fingerprinting. With this observation, UPS tries to utilize the geographical trajectory and the associated GSM power spectrogram information of a moving vehicle to identify its location with reference to a map. More specifically, this paper integrates two appealing techniques, i.e., online vehicle localization and GSM map construction. With the former, a vehicle can accurately fix its location under complex urban environments and with the latter, a reliable metropolitan-scale GSM power map can be cost-efficiently built at edges, leveraging the strong power of crowdsourcing. UPS is light-weight, requiring only a minimum hardware deployment. A prototype system was implemented to validate the feasibility of the UPS design. The results show that UPS can work stably in various urban settings and achieve an accuracy of 5.3 meters with a $90 \%$ precision, overwhelming the performance of GPS by five times.

The paper "Online Task Dispatching and Pricing for Quality-of Service-Aware Sensing Data Collection for Mobile Edge Clouds" takes the advantage of widely-distributed mobile devices, so as to provide urban monitoring-oriented crowdsourcing services by a mobile edge cloud, where fine-grained monitoring data over time are crowdsourced by mobile devices and then useful information is extracted. This paper studies the high financial cost posed on mobile devices when trying to guarantee the quality of service. This paper observed that real-world sensing data exhibit strong spatial and temporal correlations, and advanced inference methods can be employed to efficiently recover missing data. Motivated by the observation, the paper develops a near-optimal online task dispatching approach for crowdsourcing services provided by a mobile edge cloud, aiming to minimize the total cost incurred on devices while guarantee the quality of service in the meantime. Considering strategic device users with private cost information, the paper also proposes a truthful pricing policy. Extensive simulations based on real datasets show that the approach outperforms other competing schemes, producing a high quality of service with a much lower budget.

\subsection{Edge management}

The paper "Communication and Computation Cooperation in Cloud Radio Access Network with Mobile Edge Computing" studies the management of cloud radio access network (C-RAN) and mobile edge computing (MEC). Both have emerged as promising candidates for the next generation access network techniques. Unfortunately, although MEC tries to utilize the highly distributed computing resources in close proximity to user equipments (UE), C-RAN suggests to centralize the baseband processing units (BBU) deployed in radio access networks. To better understand and address such a conflict, this paper investigates the MEC task offloading control in C-RAN environments. Most prior work handling offloading control falls in the general category of resource allocation optimization. This paper focuses from the perspective of a matching problem. A model is proposed to capture the unique features in both MEC and C-RAN with respect to communication and computation efficiency constraints. The paper divides the cross-layer optimization into three stages: (1) matching between remote radio heads (RRH) and UEs, (2) matching between BBUs and UEs, and (3) matching between mobile clones (MC) and UEs. By applying the Gale-Shapley matching theory in the duplex 
matching framework, the paper proposes a multi-stage heuristic to minimize the refusal rate for user's task offloading requests. Trace-based simulation confirms that the solution can successfully achieve near-optimal performance in such a hybrid deployment.

The paper "Distributed Resource Allocation in Caching-enabled Heterogeneous Cellular Networks based on Matching Theory" studies caching management in the edge. Cache has been identified as an effective solution in reducing mobile users' (MUs') transmission delay. In order to allocate proper files into small-cell base stations (SBSs) which are close to a certain group of MUs, distributed algorithms are proposed based on matching theory in minimizing MUs' transmission latency and maximizing their satisfactions. Specifically, the paper first investigates the file allocation and MUs association problems in minimizing MUs' transmission latency, and then studies the SBSs association problem to maximize MUs' satisfactions. Moreover, the performance of the proposed algorithms is analyzed from the perspectives of stability, overhead, and complexity. Simulation results demonstrate that the proposed algorithms have a better performance compared to the benchmarks.

\subsection{Security and privacy}

The paper "Asynchronous Side Information Attack from the Edge: An approach to Identify Participants from Anonymous Mobility Traces" shows that anonymous mobility traces are vulnerable to asynchronous side information attack from the edge. This can become a critical problem since the adoption of location-based social network applications is increasing, and a large number of location traces of human mobility have been collected and published for the purpose of assisting mobile system design and scientific research. Though most mobility traces are processed before publishing by the way of replacing the true IDs and introducing noise interference. Nevertheless, the paper shows that if partial movement information is exposed to some compromised edge nodes even after the data collection period, the adversary is able to identify the participant from the anonymous mobility traces with high probability. The paper develops the attack method by exploring the accumulative temporal and spatial characteristics of individual movement. More specifically, the paper introduces $\delta$-partition to divide user locations into sub-areas, and $\epsilon$-partition to group user activities into time intervals. It is shown that a mobility trace can be uniquely represented by a set of frequent locations together with their active time intervals. The paper further derives a similarity measurement to be used by the adversary for asynchronous side information attack. Theoretical analysis was presented to prove that an anonymous participant can be correctly identied with high probability under certain condition and extensive experiments are conducted on three typical mobility datasets corresponding to the movement of bus, taxi and human, which show that the identication success ratio achieves $99 \%, 45 \%$ and $72 \%$ respectively.

\section{Summary}

This special issue was initially conceived during the annual meeting of the CCF Technical Committee of Internet (CCF TCI) at the sixth Internet Conference of China, held in August 2017, Guilin, China. The CCF TCI, CCF Transactions on Networking and conference participants all believed that edge computing and networking is a field that will have long lasting impact for the years to come. A team of guest editors was formed, representing diverse geographical regions of China, the USA, Europe, Canada, and Australia, and diverse expertise. This special issue selects papers to cover a wide range of topics, yet we believe that edge computing and networking clearly should have a wider scope. During the progress of this special issue, edge computing and networking advances fast both in academia and industry. This special issue is clearly not the conclusion, but an inception of edge computing and networking.

We are grateful to Prof. Jianping Wu and Prof. K. K. Ramakrishnan, EiCs of CCF TON who provided many suggestions in the initial stages of this special issue. We would like to sincerely thank Prof. Mingwei Xu of Tsinghua University, who provided guidance and support to each of the critical phases of the progress of this special issue. We thank Lanlan Chang and Neeraj Poduval from Springer, the reviewers and others whom we cannot list in full. Without the help from these great people, this special issue would not be possible. 